\title{
CONGENITAL ABDOMINAL WALL DEFECTS: A TOPIC REVIEW AND A CASE REPORT OF A LETHAL POLYMALFORMATION SYNDROME WITH A GIANT ABDOMINOSCHISIS
}

\author{
BARTOS V.
}

Martin Biopsy Center, Ltd., Martin

\begin{abstract}
A b s t r a c t
Abdominal wall defects (AWDs) represent a wide spectrum of congenital anomalies which are characterized by a herniation of abdominal organs through a pathologic opening in the abdominal region. The author describes a 29-year-old pregnant woman who was prenatally found to have a living female fetus with a giant omphalocele and a spinal deformation in the lumbosacral region. An evisceration of the abdominal contents into the amnionic cavity was visible. Based on USG findings the limb-body wall complex was suggested. An amniocentesis was performed and a genetic testing identified a chromosome 17q23.1-q23.2 duplication. As the fetal anomaly was considered incompatible with life, a medical termination of the pregnancy was carried out. Postmortem examination of the fetus revealed a huge abdominoschisis with a complete evisceration of the liver, stomach, small and large intestines, spleen, and the right kidney. A severe left-sided scoliosis deformity of the spine with crooked trunk was evident. The gross findings shared the features of omphalocele and limb-body wall complex with no postnatal life expectancy. Most fetal AWDs have poor prognosis. The pathology of these conditions differ greatly and require specific prenatal evaluation and pregnancy management for each entity. Accurate assessment of AWDs will allow the identification of isolated forms with better clinical outcomes compared to associated multiple malformations with chromosomal anomalies or genetic syndromes.
\end{abstract}

Key words: anterior wall defects, gastroschisis, omphalocele, limb-body wall syndrome

\section{INTRODUCTION}

Abdominal wall defects (AWDs) represent a wide spectrum of congenital anomalies which are characterized by a herniation of abdominal organs through a pathologic opening in the abdominal region $(1,2)$. The embryologic development of the ventral body wall occurs as the embryo folds in both cranial-caudal and lateral directions, changing the flat trilamellar embryonic disc into its curvilinear shape (1). This complex developmental process is well reflected in its malformations (1). There are two types of congenital defects: a) in the primaxial component and b) in the abaxial component (1). The first one is often linked to neural tube-closure defects, whereas the second one manifests as limb-body wall or ventral body wall defects (1). It is considered that an abnormal closure of the ventral body wall folds during the 4th week of development is the main cause for these entities (1). An overall prevalence of AWDs is six cases per 10,000 births (2) but it varies greatly depending on the type of malformation. The two most common types of AWDs are gastroschisis and omphalocele (1-3). Other more complex AWDs include cloacal and bladder exstrophy, ectopia cordis, limb-body wall complex, pentalogy of Cantrell, and abdominoschisis due to amniotic bands $(1,2)$. Although all of them have an AWD as a prominent feature, the presentation and prenatal and postnatal care are very different $(1,2)$. Further, many AWDs are associa-

Corresponding author: MUDr. PhDr. Vladimír Bartoš, PhD., MPH; e-mail: vladim.bartos@gmail.com (C) 2020 Bartoš Vladimír.

This work is licensed under the Creative Commons Attribution-NonCommercial-NoDerivs 4.0 License (https://creativecommons.org/licenses/by-nc-nd/4.0/) 
ted with other congenital abnormalities that have important prognostic and management implications $(1,2)$. Thus, their precise characterization during pregnancy is very important and can greatly affect outcomes. However, due to the complexity of many of these abnormalities, accurate prenatal diagnosis can be very difficult or even impossible. In the current article the author focuses on a description of three types of AWD: gastroschisis, omphalocele, and limb-body wall complex. He also describes an unusual case of a lethal congenital malformation with a giant AWD from his own practice.

\section{GASTROSCHISIS}

Gastroschisis is a paramedian AWD which is nearly always located to the right of the umbilical cord, with a normal insertion of the umbilicus (1-3). It is characterized by the herniation of the abdominal viscera outside the abdominal wall with no covering membrane or sac $(1,3)$. Typically, the bowel is eviscerated, occasionally other organs but almost never the liver $(1,2)$. The prevalence of gastroschisis is reported to be 3.09 per 10,000 births, with a live birth prevalence of 2.63 per 10,000 (1). The condition usually occurs as an isolated anomaly, but other congenital or genetic abnormalities may sometimes be found $(1,2)$. About 1.2\% of infants with gastroschisis are diagnosed as having chromosomal abberations (1) and approximately 10\% have additional somatic anomalies (1), among which central nervous system and cardiac malformations are the most common (1).

\section{OMPHALOCELE}

Omphalocele is another form of AWD represented by a midline defect located at the umbilical cord insertion site that leads to a herniation of abdominal organs into the amniotic cavity through the base of the umbilicus $(1,3)$. Unlike a gastroschisis, the eviscerated abdominal content is covered by a membrane presented by the peritoneum on the inner side, amnion on the outer side, and Wharton's jelly in between $(1,3)$. The sac of omphalocele usually contains the bowel loops and liver, or other organs such as stomach, spleen, or urinary bladder (1). The prevalence of disease is 3.29 per 10,000 births, with a live birth prevalence of 1.13 per 10,000 (1). Omphalocele is usually associated with chromosomal and non-chromosomal somatic anomalies and is frequently a component of various multiple anomaly sequences $(2,3)$. Chromosomal abnormalities have been reported in $10-12 \%$ of the neonates and $30 \%$ of the fetuses with omphalocele (4). They particularly include trisomy 18, trisomy 13, and trisomy 21 (4). Other genetic findings described are karyotypes 45X, 47XXY, and 47XXX, partial aneuploidy such as dup. (3q), dup. (11p), inv. (11), dup. (1q) and del. (1q), dup. (4q), dup. (5p), dup. (6q), del. (9p), dup. (15q), dup. (17q), Pallister-Killian syndrome with mosaic tetrasomy $12 \mathrm{p}$, Miller-Dieker lissencephaly syndrome with deletion of $17 \mathrm{p} 13.3$ and uniparental disomy 11 and 14 (4). As regards concurrent non-chromosomal somatic anomalies, they occur in $67-88 \%$ of cases (3), with cardiac and gastrointestinal defects being the most common form $(1,3)$. The finding that omphalocele can be part of a large number of unique chromosomal and genetic syndromes shows that no specific etiology has been identified for it and that AWDs may be the final pathway in a number of diverse primary developmental insults (3).

\section{LIMB-BODY WALL COMPLEX}

Limb-body wall complex (LBWC, body stalk anomaly) represents the rarest and most severe type of AWD $(1,2)$. This polymalformative syndrome includes three main features: a) exencephaly, encephalocele, or facial clefts, b) thoraco-abdominoschisis, and c) various limb defects (positional deformities, clubfoot, absent limbs) $(1,2)$. The most typical finding is a lethal massive herniation of abdominal organs in the extraembryonic coelomic cavity $(1,2)$. Severe kyphoscoliosis may also be present $(2)$. The reported prevalence of the disea- 
se is 0.12 cases per 10,000 births (including both alive and still births) $(1,2)$. Various hypotheses exist regarding the pathogenesis of LMWC. The most likely one is an abnormal lateral body folding resulting in failure of the abdominal wall development and, thus, the peritoneal cavity is open to the extraembryonic coelom (2). A failed body stalk fusion results in a short or absent umbilical cord and herniated viscera may be directly attached to the placental surface (2). Usually, there is no association with chromosomal anomalies and the recurrence rate has been demonstrated to be low (1).

\section{CASE REPORT}

A 29-year-old woman was referred to the Department of Gynecology and Obstetrics in the second trimester of her pregnancy, having found a fetus with multiple somatic malformations. A prenatal ultrasonography (USG) showed a living fetus with a giant omphalocele and a severe deformation of the spine in the lumbosacral region. A complete evisceration of the abdominal contents (liver, intestines, stomach) into the amnionic cavity was observed. A short umbilical cord was also detected. Based on the USG findings, the limb-body wall complex was suggested. An amniocentesis was performed and the mother was sent for prenatal genetic testing and consultation. A standard fetal chromosomal analysis demonstrated a normal female karyotype $(46, \mathrm{XX})$. Subsequent microarray-based comparative genomic hybridization (aCGH) identified a chromosome 17q23.1-q23.2 duplication. As the fetal anomaly was considered incompatible with life, a termination of the pregnancy was proposed after proper counseling. The mother finally opted for it. The labor was medically induced per vias naturales and resulted in a macerated dead female fetus (stage I. maceration), crown-rump length of $12.5 \mathrm{~cm}$. After the baby was born the placenta had been delivered. Both the fetus and the placenta were immediately fixed in a $4 \%$ formaldehyde solution and sent separately for a histological investigation.

A gross examination of the formalin-fixed fetus revealed a huge abdominoschisis with a complete evisceration of the liver, stomach, small and large intestines, spleen, and the right kidney (Figure 1).

Fig. 1 Ventral aspect of the formalin-fixed postmortem fetus. Giant abdominoschisis with evisceration of all abdominal organs. (L - liver, St - stomach, SI - small intestine, LI - large intestine, $\mathrm{Sp}$ - spleen, RK - right kidney)

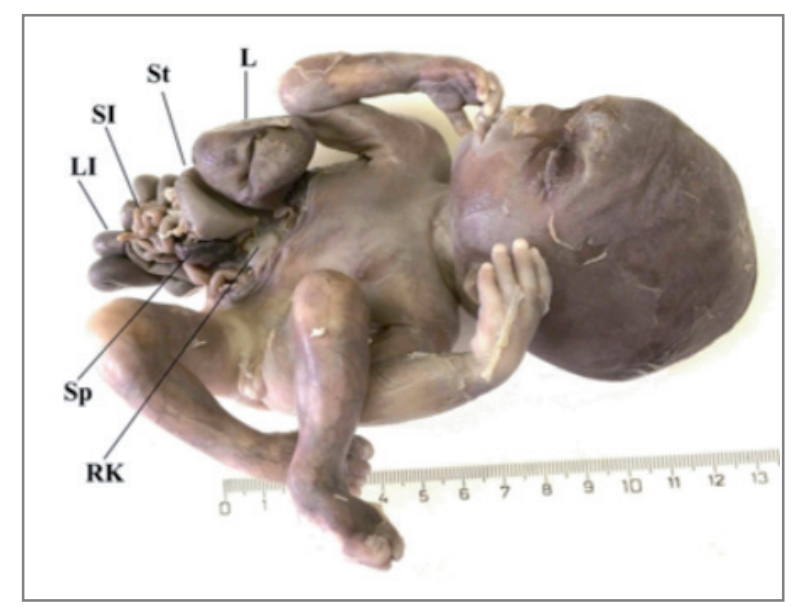

The umbilical cord stump was not found and the cord insertion site could not be identified. Further, a severe left-sided scoliosis deformity of the spine with crooked trunk was visible (Figure 2). The head and the upper and lower extremities showed no somatic malformations. Major organs in the thoracic cavity (tracheobronchial tree, lungs, heart, thymus) were normally developed (Figure 3). The placenta (size 13x10x5 cm) with membranes 
was macroscopically without substantial pathology. The umbilical cord measured $8 \mathrm{~cm}$ and contained two umbilical arteries and one umbilical vein. An overall microscopic assessment of the taken organs (brain, lungs, heart, tymus, liver, spleen, both kidneys and adrenals) was limited due to the tissue autolysis, but no convincing histopathological changes seemed to be present. The gross findings of the fetus corresponded with the prenatally diagnosed severe congenital malformation with no postnatal life expectancy.

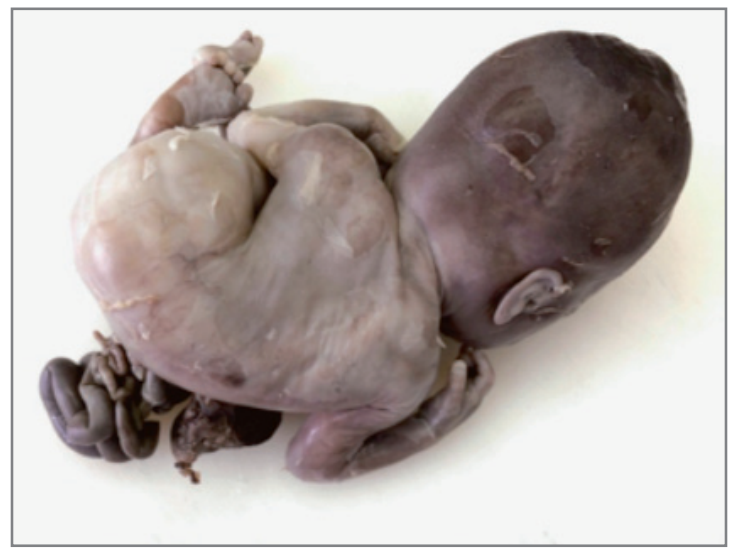

Fig. 2 Dorsal aspect of the formalin-fixed postmortem fetus. Severe scoliosis deformity of the trunk.

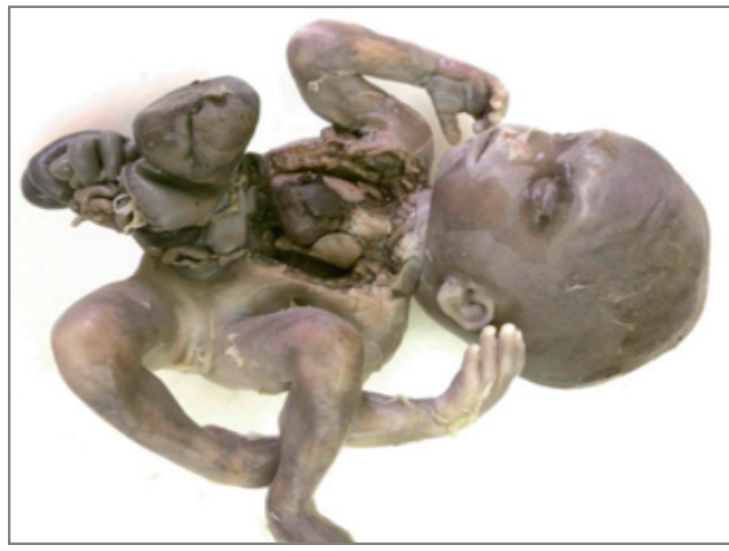

Fig. 3 Ventral aspect of the formalin-fixed postmortem fetus after opening the chest wall.

\section{DISCUSSION}

The author presents a unique case of a congenital polymalformation syndrome accompanied by a giant AWD with evisceration of all abdominal organs. This lethal condition could not be specifically classified, but it shared the features of omphalocele and LBWC. Both of these units have also been considered prenatally by USG. The exact pathogenesis of omphalocele is still a matter of debate. It may represent the failure to return of the bowel loops after the physiological herniation from the 6th to 10th week post-fertilization when the fetal midgut extends into the extraembryonic celom, occupying the proximal segment of the umbilical cord (1). The physiological hernia seldom exceeds $7 \mathrm{~mm}$ in diameter or rarely persists after 12 weeks of gestation when the midgut returns into the abdominal cavity (1). Other pathogenic theories include a failure of the process of body in-folding, that occurs at 3-4 weeks of gestation (3). During embryonic development three areas of body in-folding occur simultaneously and each is associated with a distinct type of omphalocele (3). Cephalic folding defects result in a high or epigastric omphalocele as seen in the pentalogy of Cantrell (3). Lateral folding defects result in the "classic" omphalocele with a midabdominal defect (3) and caudal folding defects result in a low or hypogastric omphalocele as seen in bladder or cloacal extrophy (3). In our case a combination of a huge abdominoschisis with a severe spinal deformity suggested a possible LBWC. Although the fetus did not manifest apparent malformations of the limbs, this diagnosis might be considered. An interesting feature in our case was a chromosome 17q23.1-q23.2 duplication, which could teoretically support an evidence of LBWC. It is known the candidate gene within the duplicated region is TBX4, a T-box transcription factor required for a normal hind limb development $(5,6)$. The duplication of chromosome 17q23.1-q23.2 has been particularly associated with congenital clubfeet $(5,6)$, but even with other lower limb skeletal malformations, e. $g$. hip dysplasia $(5,6)$, broad and shortened metatarsals and calcanei, small distal tibial epiphyses, and thickened ischia (6). In a study of 25 fetuses with LBWC (7) 96\% of them had 
limb defects, with the lower limbs being affected more frequently. Among those anomalies the clubfoot was one of the most common (44\% of cases). It has been documented (5), the chromosome 17q23.1-q23.2 duplication is characterized by a very variable expressivity and incomplete penetrance. Peterson et al. (5) observed that some affected individuals had severe manifestations, while others had none at all. Therefore, in our case, a limb anomaly may not have manifested. Anyway, this case is unique because to the best of the author's knowledge no association of the chromosome 17q23.1-q23.2 duplication with either omphalocele or LBWC has been published until now.

\section{CONCLUSION}

Fetal abdominal wall defects represent a complex group of congenital conditions with a broad spectrum of manifestations. Many of them are associated with chromosomal and multisystem structural anomalies that worsen the prognosis and the final outcome. Prenatal recognition of the more complex forms of abdominal wall defects is crucial for appropriate parental counseling and further pregnancy management.

Acknowledgement: The author greatly thank MUDr. Ivana Paučinová (Department of Genetics, Faculty Hospital in Žilina) for valuable clinical information.

\section{REFERENCES}

1. Drăgusin RC, Sorop-Florea M, Pătru CL, et al. Fetal abdominal wall defects. In: Tudorache S. Congenital anomalies: From the embryo to the neonate. IntechOpen 2018:207-230.

2. Peterson JF, Ghaloul-Gonzalez L, Madan-Khetarpal S, et al. Familial microduplication of 17q23.1-q23.2 involving TBX4 is associated with congenital clubfoot and reduced penetrance in females. Am J Med Genet A 2014;164A(2):364-9

3. Alvarado DM, Aferol H, McCall K, et al. Familial isolated clubfoot is associated with recurrent chromosome 17q23.1q23.2 microduplications containing TBX4. Am J Hum Genet 2010;87(1):154-160.

4. Pakdaman R, Woodward PJ, Kennedy A. Complex abdominal wall defects: Appearances at prenatal imaging. Radiographics 2015;35(2):636-649.

5. Wilson RD, Johnson MP. Congenital abdominal wall defects: an update. Fetal Diagn Ther 2004; 19(5):385-98.

6. Van Allen MI, Curry C, Walden C, et al. Limb body wall complex: II. Limb and spine defects. Am J Med Genetics 1987;28(3):549-65.

7. Chen CP. Chromosomal abnormalities associated with omphalocele. Taiwan J Obstet Gynecol 2007;46(1):1-8.

Received: September, 3, 2020

Accepted: October, 12, 2020 